\title{
An Invitation to Join the Consortium on Thyroid and Pregnancy
}

\author{
Tim I.M. Korevaar ${ }^{a}$ Peter N. Taylor ${ }^{b}$ Colin M. Dayan ${ }^{b}$ Robin P. Peeters ${ }^{a}$ \\ a Department of Internal Medicine and the Rotterdam Thyroid Center, Erasmus University Medical Center, \\ Rotterdam, The Netherlands; ${ }^{b}$ Thyroid Research Group, Institute of Molecular and Experimental Medicine, \\ School of Medicine, Cardiff University, Cardiff, UK
}

Dear Editor,

The clinical evidence highlighting the importance of adequate maternal thyroid function during pregnancy for an optimal pregnancy outcome is rapidly accumulating. However, due to large differences across studies regarding study populations and outcomes, it is difficult to generalize all available evidence and to provide evidence-based recommendations. Furthermore, many important clinical questions remain unanswered because single studies are not adequately sized to study small subgroups and clinical threshold effects.

In order to overcome these limitations, we have founded the Consortium on Thyroid and Pregnancy. The goal of this international collaboration is to create a formal platform for cooperation between researchers and cohorts that facilitates highquality studies on the association of gesta- tional thyroid function with adverse pregnancy outcomes. The initial aim of this collaboration is to study the association of maternal thyroid function and autoimmunity with preterm birth and birth weight, by performing individual participant-based meta-analyses combining data from prospective cohorts across the globe.

Currently, the Consortium consists of 23 studies with data on more than 80,000 mother-and-child pairs from across the globe. We are performing systematic searches to identify eligible studies or datasets from the published literature that can be included in our current project.

Via this letter, we would like to invite anyone that has data available (published or unpublished) that match the inclusion criteria (see below) to join this novel consortium and to contribute to advancing ev- idence-based medicine within the field of thyroid function and pregnancy.

Inclusion criteria for the current project:

- Nonselected or population-based prospective cohorts.

- Measurements of TSH, free T4 or thyroid antibodies in pregnant women (any gestational age).

- Complete follow-up until the end of the pregnancy.

If you wish to participate, or require more information, please contact us via email.

\section{Disclosure Statement}

The authors declare that no financial or other conflict of interest exists in relation to the content of the article.

\begin{tabular}{|c|c|}
\hline KARGER & $\begin{array}{l}\text { (๑) 2016 European Thyroid Association } \\
\text { Published by S. Karger AG, Basel }\end{array}$ \\
\hline $\begin{array}{l}\text { E-Mail karger@karger.com } \\
\text { www.karger.com/etj }\end{array}$ & $2235-0640 / 16 / 0054-0277 \$ 39.50 / 0$ \\
\hline
\end{tabular}

Tim I.M. Korevaar

Department of Epidemiology and Endocrinology, Erasmus Medical Center Room Na-2913, Postbus 2040

NL-3000 CA Rotterdam (The Netherlands)

E-Mail t.korevaar@erasmusmc.nl 